\title{
A ROBOT TEST-BED FOR ASSISTANCE AND ASSESSMENT IN PHYSICAL THERAPY
}

\author{
Rahul Rao, Sunil K. Agrawal ${ }^{\mathrm{ii}}$, John P. Scholz ${ }^{\mathrm{iii}}$ \\ Mechanical Systems Laboratory \\ University of Delaware, Newark, DE 19716.
}

\section{Abstract}

This article describes an experimental test-bed that was developed to assist and assess rehabilitation during physical and occupational therapy. A PUMA 260 robot was used for which a controller and interface software was developed in-house. The robot can operate in two modes: (i) passive and (ii) active. In the passive mode, the robot moves the subject's arm through specified paths. In the active mode, a subject guides the robot along a predefined path overcoming a specified joint stiffness matrix. In this mode, the controller provides gravity compensation so that the robot can support its own weight in an arbitrary configuration. The developed graphical interface enables display of the current configuration of the robot in real-time, customize experiments to a specific subject, and collect force and position data during an experiment. The results of a preliminary study using this test-bed are also presented along with issues involved in choice of paths and interpretation of the results.

Keywords: Robot, Rehabilitation, Assessment, Physical Therapy.

\section{Introduction}

Active exercise is an important component of rehabilitation. Resistance is typically accomplished by using expensive exercise equipment or is applied manually by a therapist. Most available exercise equipment allowing for controlled application of forces to a limb or the trunk limit motion to one plane or forces are applied directly on to a single joint. As such, their relevance to functional movements is extremely limited. And although manual resistance applied by a therapist allows for exercise of multiple degrees-of-freedom (Voss et al., 1985), it requires the therapist's complete attention to only one patient at a time, increasing the cost of treatment.

The need for objective, quantitative and reliable evaluation tools to assess the neuromuscular performance of patients is critical to both physical and occupational therapy (Carr and Shepherd, 1990; Chandler et al., 1980). The ability to quantify movement performance has been a particular problem in these disciplines. This is specially the case in neurological rehabilitation, where most assessments 
of motor function have been based on an ordinal scale of quantification (Bayley, 1935; Poole and Whitney, 1988; Rothstein, 1985; Scholz, 1993).

These facts indicate that the development of a device that would allow for controlled motion of the entire limb in quasi-functional patterns could improve patient evaluation and treatment effectiveness while reducing its time and cost. Some important issues that need to be addressed are (i) development of a user friendly robot with a safe control system, (ii) development of a versatile subject interface, and (iii) design of suitable experiments to evaluate the effectiveness of the approach. However, there have been only a handful of studies that have attempted to develop complex machines to accomplish this task and that have evaluated protocols for their application.

Noritsugu et al. (1996) developed a two degree-of-freedom rubber artificial muscle manipulator and performed experiments to identify human arm parameters. Impedance control has been suggested as an effective approach to control human-machine systems (Hogan, 1985) and has been studied for direct drive robots (McKormic and Schwartz, 1993). Some preliminary studies have been presented on the application of robot technology to enhance the rehabilitation of stroke patients (Krebs et al., 1995). These studies suggest that robots are promising new tools in this area. A prototype for bimanual lifting
(Lum et al., 1995) and MIME (Mirror Image Motion Enabler) have been reported for post stroke therapy (Lum et al., 1997).

This article presents some recent efforts at University of Delaware in the development of a robot test-bed to assist and assess rehabilitation. The salient features of this study are: (i) an in-house developed controller for the robot motivated by safety considerations, (ii) a versatile interface that can be used to customize subject experiments, (iii) a mechanism to collect force and position data during an experiment, (iv) protocols to provide assessments using the robot test-bed. The outline of this article is as follows: Section 2 presents a description of the robot set-up. The design of experiments, data analysis, and results are described in Section 3. These are followed by a discussion of the results, their implications and conclusions.

\section{Robot Test-bed}

The test-bed consists of a six degreeof-freedom PUMA Mark II 200 series robot arm. Due to inherent limitations of the original controller provided by the manufacturers, an in-house controller was developed that uses LM628 based servo controllers interfaced with a Pentium $233 \mathrm{MHz}$ computer. The computer also handles the user interface and real-time display of the graphics. A schematic of the setup is shown in Figure 1 along with data flow in the system. The robot joints are equipped with optical encoders that 
provide a resolution of roughly 0.005 degrees and a 6-axis force-torque sensor, manufactured by JR3 Inc. (Model No. 67M25A). Even though the robot has the capability to move in 3dimensional space, in this study, the robot motion was restricted to the vertical plane.

The software for the robot was written in an object-oriented environment. Some of its special features are: (i) ability to interact with other applications such as MATLAB, (ii) personalized and flexible experiments through an interactive user interface, (iii) a two-dimensional graphic visualization of the robot motion on the monitor. The software allows the robot to run in two modes: (i) Passive (P) and (ii) Active (A). In the P-mode, the on the movement of the robot by the subject. This mode is also effectively used before experimentation in Amode, described later.

A typical session in the P-mode has the following features:

- Locate 40 points on the computer screen, 20 each on the inner and outer walls of a tunnel containing the path. Alternatively, a path defined earlier or stored in the computer can be recalled for a current use. Typical paths created using this procedure are shown in Figure 2.

- During path execution, the software draws the inner and outer walls and locates 20 discrete points along the central line between the walls.

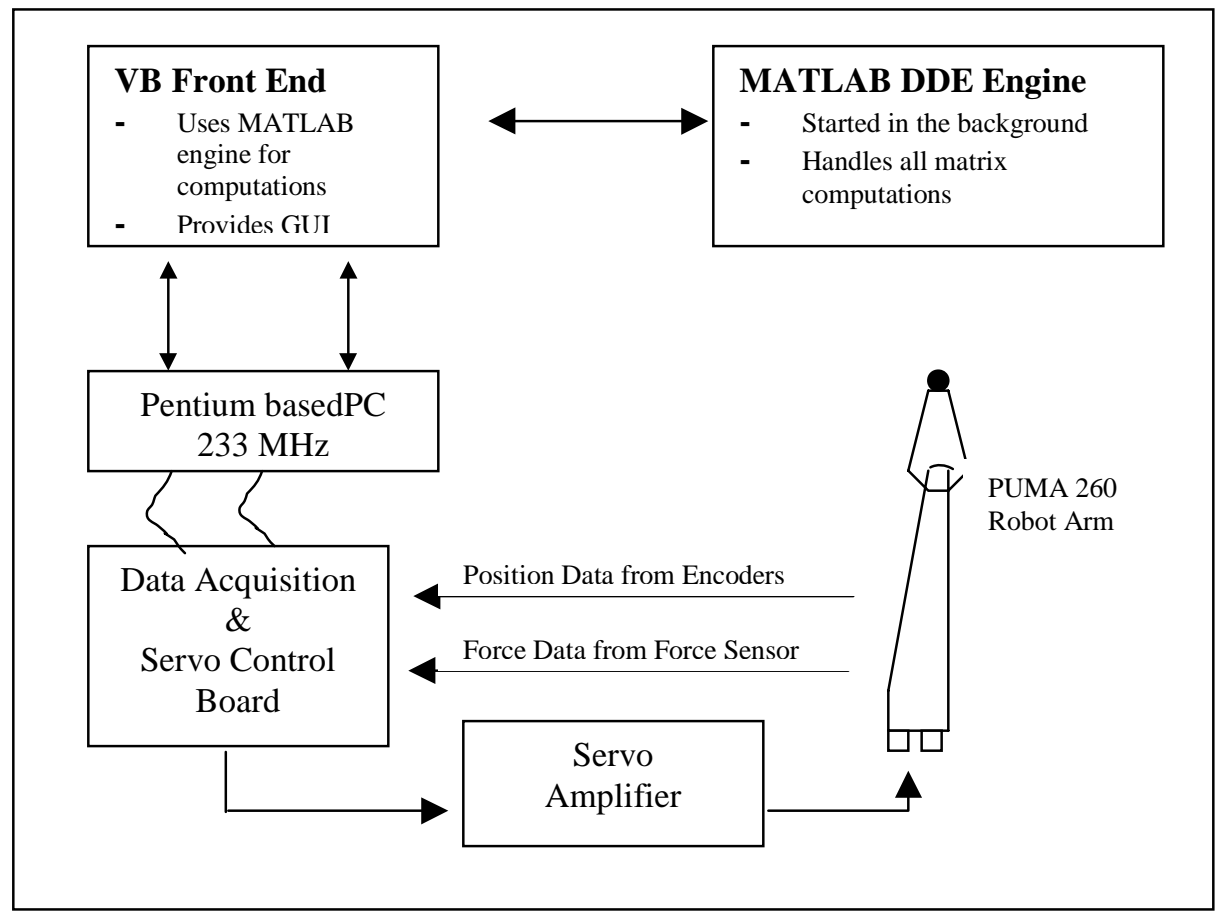

Fig. 1: A schematic of the modules in the system along with flow of data

robot moves the subject hand within the workspace, with little or no control
These points are then utilized to solve the inverse kinematics 
between two successive points and the robot tracks the central line by moving between two successive points. The subject is instructed to lightly hold the robot end-effector during motion of the robot.

A typical session in the active mode is:

- The therapist or experimenter recalls a path or defines a new path by describing 40 points on the outer and inner walls. The robot moves the subject to the starting point of the central line and handles the control over to the subject.

- The subject is in full control of the robot arm and makes an attempt to track the central line while overcoming the stiffness specified at the joints of the robot. The stiffness can be varied along the path using control panels on the screen.

- During motion, the position of the end-effector and subject exerted forces and moments are recorded by the 6 DOF force sensors.

- During experiment, if the subject hits a wall boundary, the robot temporarily takes over control, moving the handle/hand back to the nearest point on the center line, and then returns control to the subject. The color of the wall that is hit changes during this period giving the subject a visual cue of the collision. The original color is restored once the robot end is at the central line and the control is handed over to the subject. A trial gets completed when the subject traverses the path in both forward and reverse directions, although more repetitive trajectories can be specified in principle.

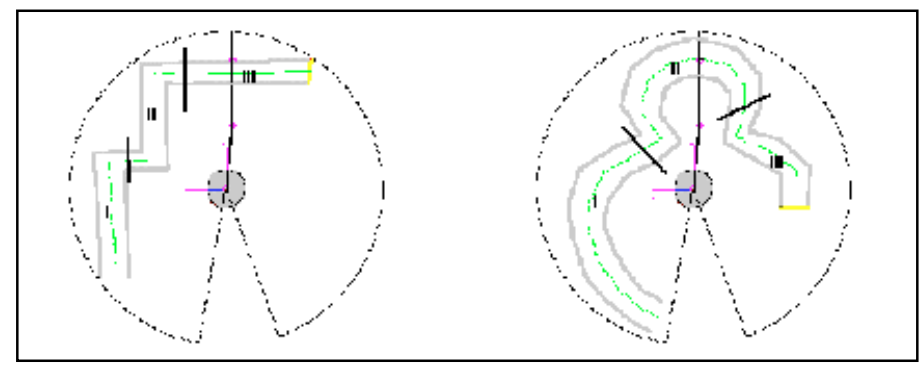

Fig. 2: Typical paths created by the software divided into regions.

Since PUMA 200 robot is heavy, in a general configuration, the links will fall under their own weight. To alleviate the subject from working against this gravity load, a scheme was developed to gravity balance the robot by providing actuator torque appropriate to the configuration of the robot in the plane. A gravity model for the robot in the vertical plane was developed using analytical approach verified by experimental data (Rao, 1999). It was observed that this model for the gravity loading worked quite well over the useful workspace of the robot. The geometric planning for the robot was done using its inverse kinematic model.

\section{Experimental Studies}

\subsection{Selection of Paths}

In this exploratory study, experiments were conducted on four healthy adult subjects. In order to understand the role of paths during experimentation, two 
paths $A$ and B, shown in Figure 2, were used. Path A consists of linear segments while path B consists of circular segments, with both intermixed with sharp turns. The rationale for choosing these two paths is that an arbitrary path can be constructed using combinations of these two. Each of these paths was divided into 3 regions. This was done to observe if any of the regions had the feature of being particularly easy or difficult to negotiate. Each path was traversed forward and backwards and we label this as a block of experiment. Four blocks of experiment were completed on each path.

The first three blocks had identical experiment conditions. In the fourth block, joint stiffness were enhanced by a factor of 2. This was done to observe how learning during the first three blocks of experiments helps a subject overcome enhanced stiffness during the fourth block of experiments. Certain factors were kept consistent across blocks of experiments and across subjects. These were:

A reminder to the subject before each block of experiment about experiment objectives, i.e., to remain within the two walls on the screen and track the central line as closely as possible.

A reminder to the subject to maintain constant speed during the entire study. Each subject was given two practice trials in the active mode to facilitate determining a comfortable speed for the experiments.
Standardizing their grip on the endeffector so that their elbow points straight ahead and they have a clear view of the monitor.

The collected data consists of the following information: region of the path, $\mathrm{X}$ and $\mathrm{Y}$ co-ordinates of the end point, $\mathrm{X}, \mathrm{Y}, \mathrm{Z}$ forces and moments. This data was was analyzed off-line using MATLAB. The hardware allowed us a sample rate of roughly $1000 \mathrm{~Hz}$.

\subsection{Data Analysis:}

The central line was defined for convenience as the intended path for the experiments. Deviations from the central line $d$ provided indicators of a subject's performance and consistency. Position data analysis was conducted for all four blocks of experiments.

The fundamental difference between position data analysis and force data analysis is that there is no intended or known ideal force trajectory with which a comparison can be made. Further, even though subjects attempt to maintain a constant speed in the trials, they are not able to achieve it exactly. This leads to a different number of data samples collected in each trial. Thus, in order to bring all subjects to a common time base, a normalization procedure was employed which included an interpolation between elements of each column in the data array. This interpolation was performed using cubic splines, resulting in a new array consisting of 
the normalized elements. The algorithm for the analysis of subject data within a block of experiments can be summarized as follows:

For each trial in a block, isolate samples belonging to regions 1,2 and 3 into different arrays.

Normalize elements of each array to obtain normalized values of the samples.

Compute the signed distance of the end-effector from the central line for each sample.

Concatenate all normalized samples that belong to a certain region within a particular block of four trials.

Identify samples at every $5 \%$ of the total number of samples for each region. Compute the mean and standard deviation of the samples and obtain a graphic representation of the variation in a particular region of a path during a block of experiments.

\subsection{Results}

Because of the preliminary nature of these tests, all data collected during the experiments were analyzed visually. Among these, the deviation $d$ and $\mathrm{z}-$ moment from the force sensor $\mathrm{Mz}$ showed some trends and were therefore analyzed in greater detail. Figures 3 and 4 show a set of four plots that represent the normalized mean deviations for a subject tracking the central line in a particular region of the path. These plots are shown for all three blocks of experiments. The eight plots in the two figures represent a general trend among all subjects in the experiments. Across the three blocks, one can observe a decrease in the mean distance from the center path, accompanied by a decrease in the variable error band about this mean distance. This indicates that a subject was able to track the center more consistently as more experiments were conducted.

Table 1: Ferformance details for all subjects, S 1 through S 4

\begin{tabular}{|c|c|c|c|c|c|c|c|c|c|c|c|c|}
\hline \multicolumn{13}{|c|}{ Hits on the walls while traversing path $\mathrm{A}$} \\
\hline & \multicolumn{4}{|c|}{ Region I } & \multicolumn{4}{|c|}{ Region II } & \multicolumn{4}{|c|}{ Region III } \\
\hline Block & S 1 & S 2 & S 3 & S 4 & S 1 & S 2 & 83 & 84 & S 1 & S 2 & S 3 & 84 \\
\hline 1 & 2 & - & 1 & - & - & - & 4 & - & - & - & 1 & - \\
\hline 2 & 1 & - & - & - & 1 & - & - & - & - & - & - & - \\
\hline 3 & - & - & - & - & - & - & - & - & - & - & - & - \\
\hline 4 & - & - & 2 & - & 4 & - & 5 & - & 2 & - & - & 2 \\
\hline
\end{tabular}

Table 2: Ferformance details for all subjects, S 1 through S 4

\begin{tabular}{|c|c|c|c|c|c|c|c|c|c|c|c|c|}
\hline \multicolumn{13}{|c|}{ Hits on the walls while traversing path B } \\
\hline & \multicolumn{4}{|c|}{ Region I } & \multicolumn{4}{|c|}{ Region II } & \multicolumn{4}{|c|}{ Region III } \\
\hline Block & S 1 & S 2 & S 3 & $\mathrm{~S} 4$ & S 1 & 52 & 83 & S4 & S 1 & S 2 & 83 & 84 \\
\hline 1 & - & - & 3 & - & - & - & 5 & - & 1 & - & 2 & - \\
\hline 2 & - & - & - & - & - & - & - & - & - & - & - & - \\
\hline 3 & - & - & 1 & - & - & - & - & - & - & - & - & - \\
\hline 4 & - & - & 1 & 2 & - & - & 5 & - & - & - & - & 3 \\
\hline
\end{tabular}



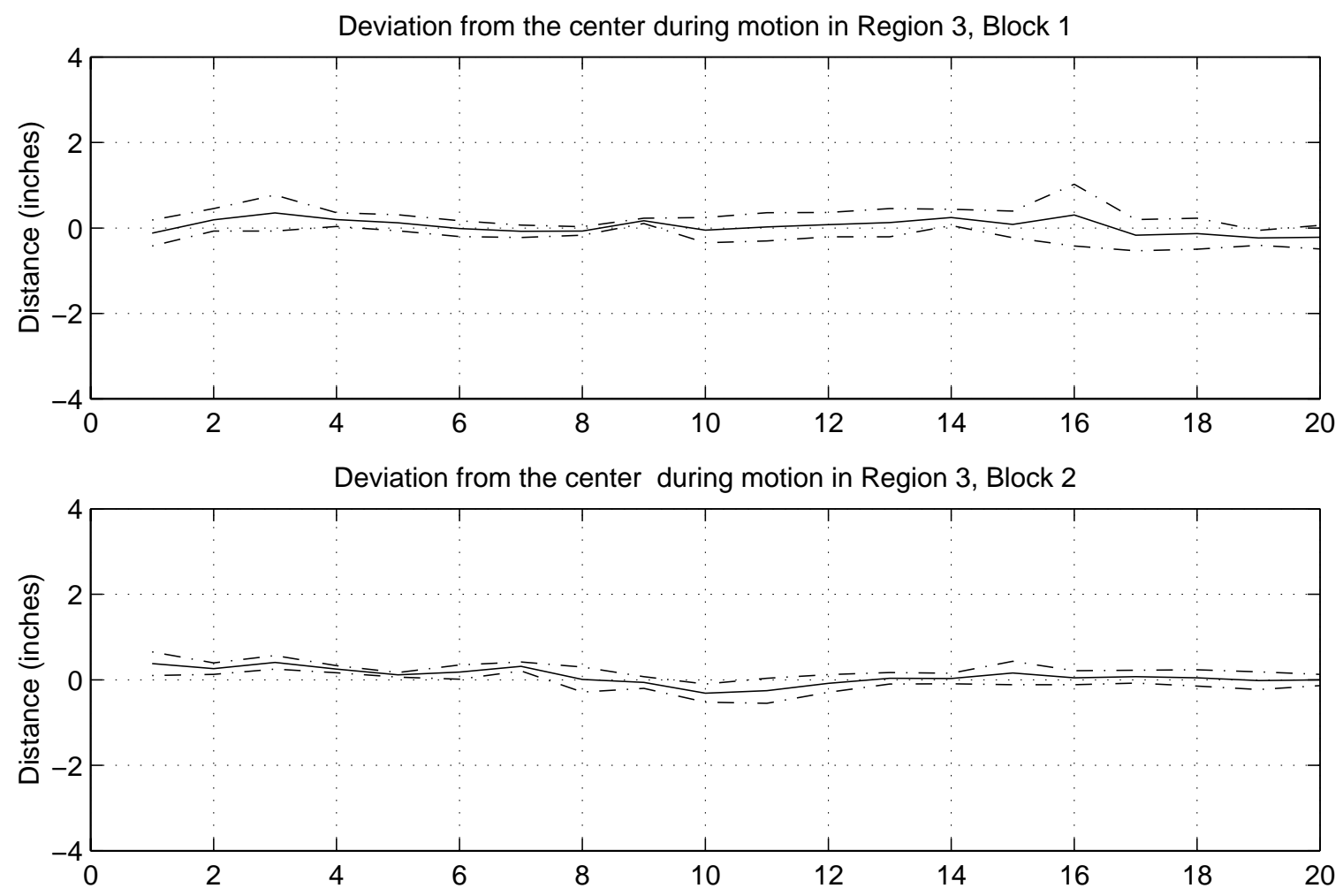

Deviation from the center during motion in Region 3, Block 3

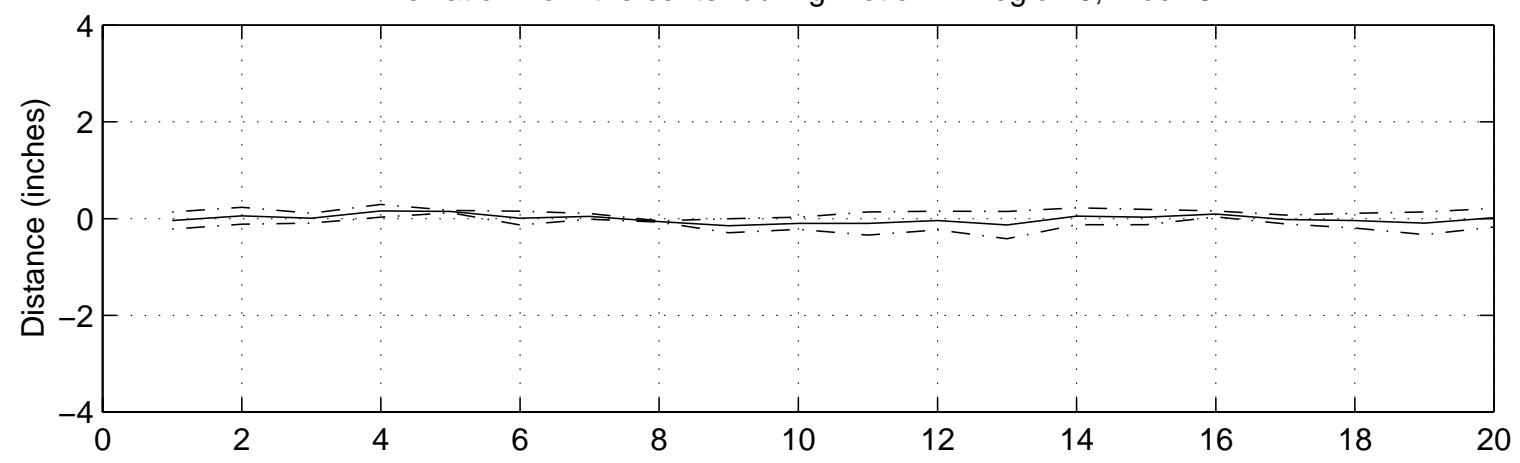

Deviation from the center during motion in Region 3, Block 4

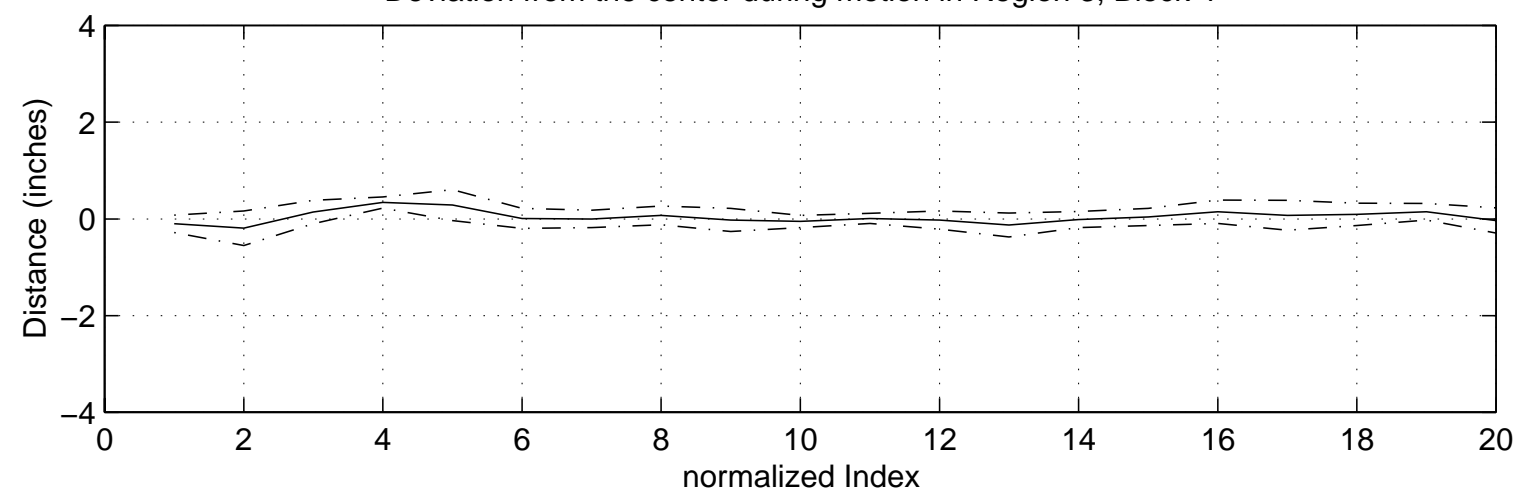

Fig. 4 Distance from the center line for subject 1, region 3, path B 

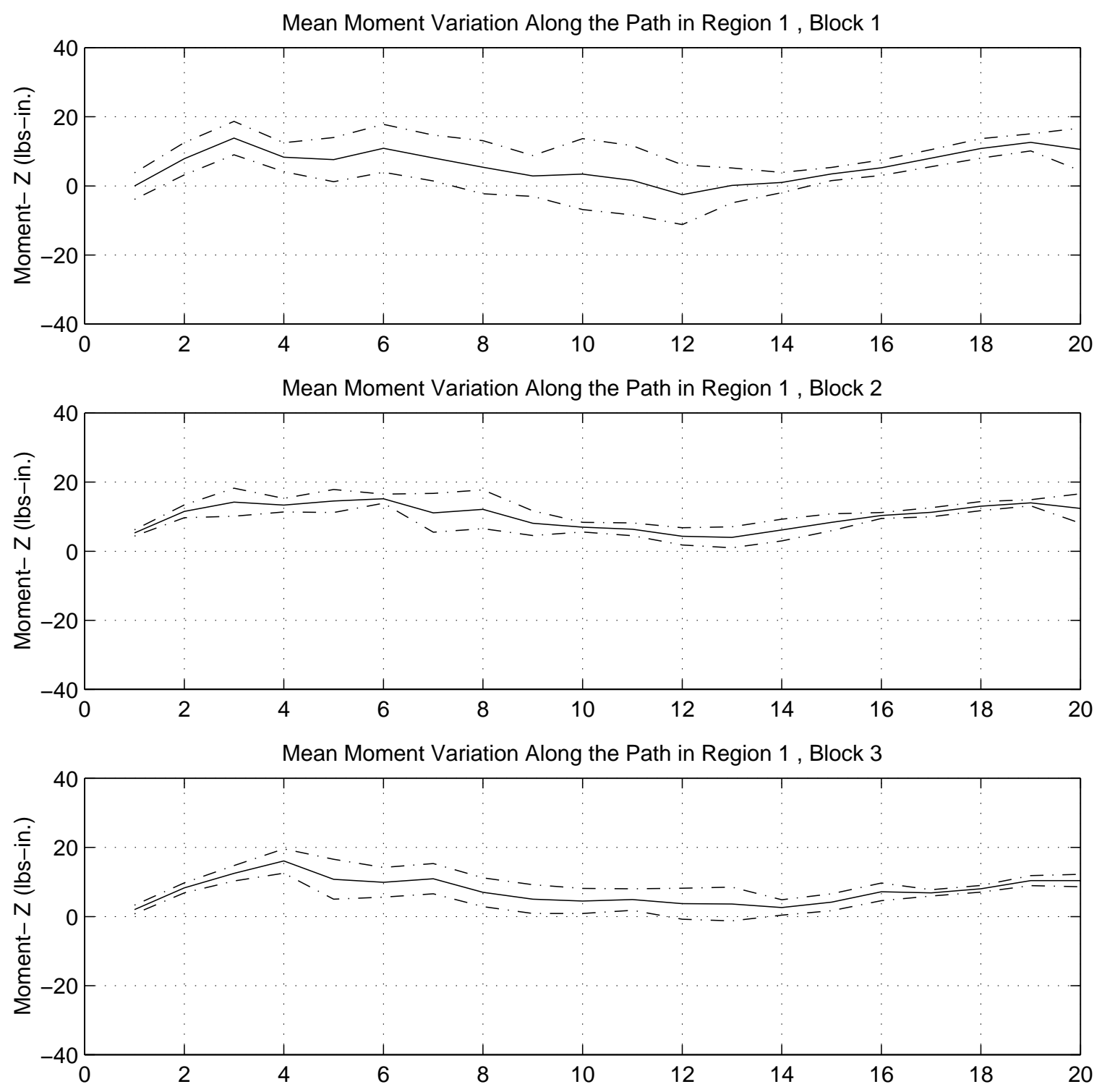

Mean Moment Variation Along the Path in Region 1, Block 4

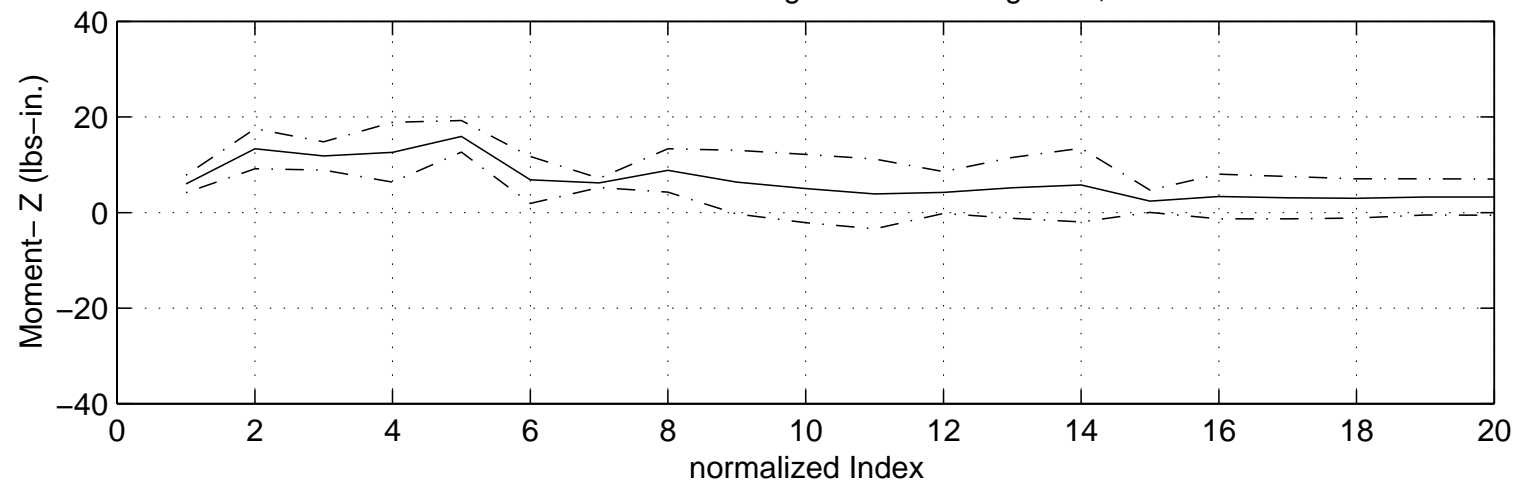

Fig. 5 Moments about the $\mathrm{Z}$ axis for subject 4, region 1, path $\mathrm{A}$ 

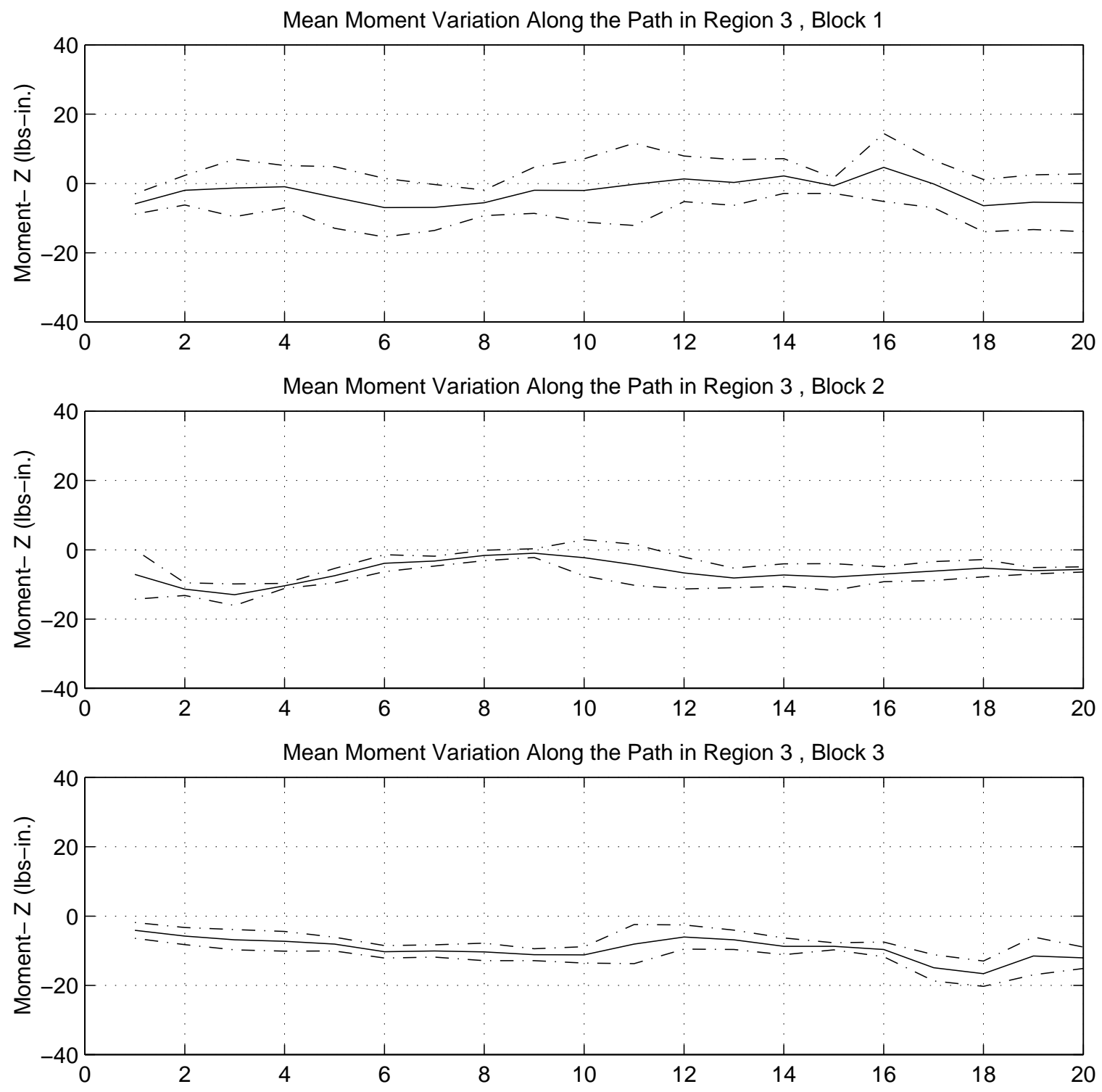

Mean Moment Variation Along the Path in Region 3 , Block 4

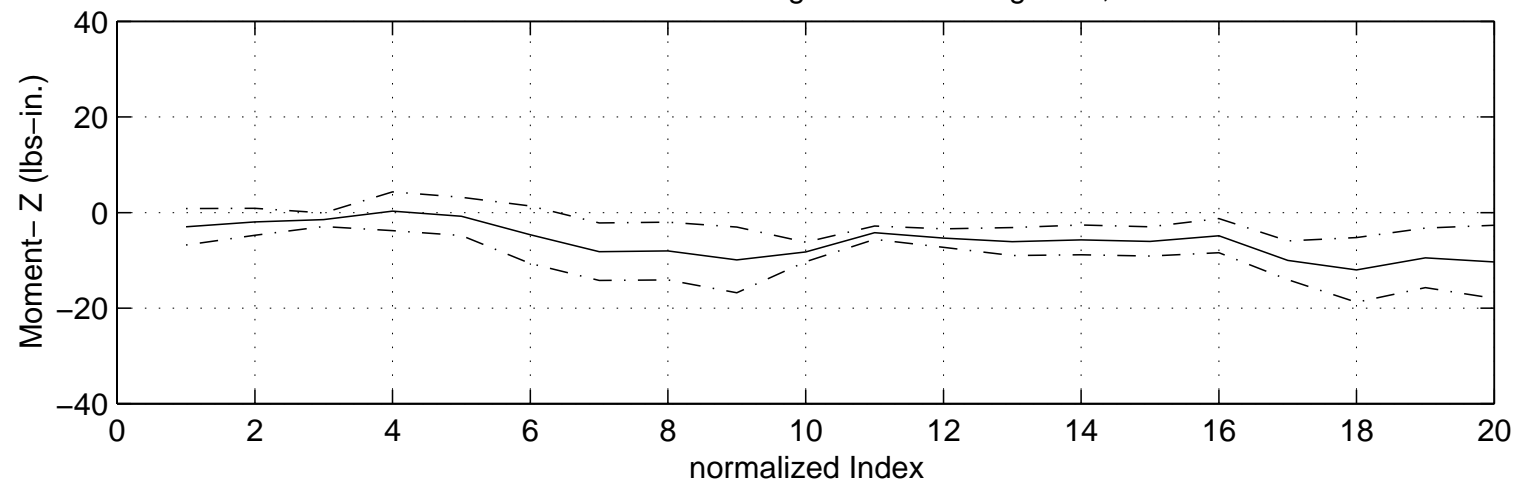

Fig. 6 Moments about the $\mathrm{Z}$ axis for subject 1, region 3, path $\mathrm{B}$ 
As far as hits on the wall were concerned, Tables 1 and 2 reveal that there were fewer hits on the walls in block 3 compared to block 1, although subjects hit the wall infrequently nonetheless.

From the plots representing the moment about the $\mathrm{Z}$ axis (Figure 5 and 6 ), perpendicular to the plane of motion, one can observe that the profile of $\mathrm{Mz}$ becomes smoother across blocks of experiments. This general trend suggests that a subject learned to traverse the path with fewer jerks, or more smoothly across blocks as more experiments were conducted, although a more detailed analysis is clearly needed.

This study indicates that some regions of the two paths $\mathrm{A}$ and $\mathrm{B}$ enabled a better performance by some subjects as opposed to the others, but the trends were not similar across subjects.

\section{Discussion}

This article has described the design and fabrication of an experimental testbed consisting of a PUMA 260 robot arm with an in-house designed controller unit, interfaced with a Pentium based computer. The software is written in an object oriented environment with a graphical user interface that enables one to customize experiments for a subject. The software also provides the user with a real time animation of the robot motion and the path traced by the robot endeffector. The optical encoders at the robot joints provide position data while a six degree-of-freedom force-torque sensor at the end-effector provides force and torque data that can be used to assist and quantify patient rehabilitation.

Our test-bed provides a means to measure quantitatively the performance of quasi-functional movement patterns by patients with a variety of movement disorders. A significant problem in patients who have suffered a stroke, for example, is the presence of coordination deficits. These are especially difficult to quantify. Although information obtained about movement patterns produced by the end-effector (i.e., hand or foot) does not provide detail about individual impairments, the information provided may be extremely valuable for assessing the effects of specific impairments or different levels of impairment on functional movement patterns. With our test-bed, quantitative assessment of quasifunctional movement patterns is made possible where such information was previously very difficult to obtain. Recent research has indicated that movement trajectories may be planned by the nervous system in terms of movement of the end-effector rather than the individual movement components (Flash and Hogan, 1985; Hogan and Winters, 1990; Hogan, 1995; Scholz and Schoner, 1999). Such information may be essential, therefore, for identifying deficits in central planning or the transformation 
of a central plan into action. Most importantly, the information provided will be helpful in customizing a patient's treatment, for helping to determine when to stop treatment because it is yielding no further improvement, and for providing data to evaluate the efficacy of particular treatment approaches. Combining information obtained from our test-bed with other types of data, e.g., video analysis of joint motion and/or electromyography, should provide a means for assessing the relationship between whole limb motion and the underlying impairments. In our very preliminary tests of the device, we have shown that information on end-effector position and force can be obtained which may be useful for characterizing changes in performance.

The ultimate goal of rehabilitation is to improve the patient's functional capabilities, regardless of the underlying pathology. Our test-bed can potentially provide a number of advantages for neuromuscular rehabilitation. For example, when there is weakness of many muscles that act to control movement and stability of a limb, strength training of each of these muscles is necessary. The use of single degree-of-freedom dynamometers to train the affected muscles can be very time-consuming. Our device, on the other hand, would allow for simultaneous strength training of many muscles through the performance of quasi-functional patterns of movement. Although free weights or pulley systems allow for simultaneous strength training of many muscles as well, it may be impossible for a patient to control free weights in the early stages of rehabilitation. Moreover, because our device can, in principle, be made to provide accommodating resistance throughout the range of motion, a patient would never work against more resistance than he or she can handle.

By providing real-time animation of robot motion and movement constraints, our test-bed provides a means for providing immediate feedback to the patient about the results of their movement along a specified spatial path (e.g., patient keeps the hand centered, deviates toward the outer wall, etc.), which may be made simple or complex according to the current abilities of the patient. In addition, more performance oriented feedback can be provided to the patient after one or several trials (e.g., the force field generated by the hand during the movement). Such information is essential for motor learning (Weinstein, 1990). Ultimately, our goal is to use the graphics interface to make therapy game-like for the patient with the goal of increasing patient interest and motivation.

Most functional tasks involve movement of an entire limb or a substantial number of joints at the very least. It is also common for such tasks to be carried out in all three spatial 
dimensions simultaneously. An important goal, therefore, is to design training paradigms that approximate as closely as possible this reality. The tests reported in this article evaluated movement of the entire upper extremity, although the movements were limited to a single plane. Thus, a important future direction will be to extend the development of the robot's use to three-dimensional movements. This will require more complicated graphic displays to provide the patient with convincing information about the hand's position in three-dimensional space. However, it will first be important to improve the robot's performance in the current set-up and to perform more quantitative tests of its performance with human subjects, including patients with movement deficits.

Although the Puma robot was designed for industrial use, we have shown that it has potential for use in rehabilitation as well. However, several problems will need to be resolved before this particular robot can be used effectively with patients. Currently, we are working to improve the interface of the robot handle with the subject's hand so that it can be accommodated to the different grasping abilities of patients. This is a general problem faced with the use of any robot, however. In terms of controlling forces applied to a subject's hand, it would be ideal to be able to specify the Cartesian stiffness at the end-effector rather than a matrix of joint stiffness. To date, this has been difficult because of difficulty in characterizing and accounting for joint friction. This problem does not preclude the robot's use for quantifying movement deficits or in training movement patterns, although it may limit its overall usefulness.

The most encouraging result of our work to date has been the development of a graphical user interface that is flexible and easy to use. As described in the Results section, subjects learned to minimize deviations from the center line in repeated trials. Also, the torque they applied to the end-effector became smoother over blocks of experiment. These results suggest that robot set-ups like these possess the potential of providing effective aids for rehabilitation.

Acknowledgments: The authors acknowledge support of National Science Foundation Presidential Faculty Fellowship during the course of this work.

\section{References}

Bayley, N., The development of motor abilities during the first three years. Monographs of the Society for Research in Child Development, 1, 126, 1935.

Carr, J.H. and Shepherd, R.B., A Motor Relearning Programme for Stroke, Rockville, MD: Aspen Publishers, Inc., 1990. 
Chandler, L.S., Andrews, M.S. and Swanson, M.W., Movement Assessment of Infants - A Manual. Rolling Bay, Washington, 1980.

Flash, T. and Hogan, N., The coordination of arm movements: an experimentally confirmed mathematical model, Journal of Neuroscience, 7, 1688-1703, 1985.

Hogan, N., Impedance Control: An Approach to Manipulation, parts I, II and III, ASME Journal of Dynamic Systems, Measurement and Control, Vol 107, pp 1- 24, 1985.

Hogan, N., The mechanics of multijoint posture and movement control, Biological Cybernetics, 52, 315-331, 1985.

Hogan, N. and Winters, J.M., Principles underlying movement organization: upper limb. In J.M. Winters and S.L-Y. Woo [Eds.]. Multiple Muscle Systems: Biomechanics and Movement Organization, pp. 182-194. New York: Springer-Verlag, 1990.

Kazerooni, H., On the Robot Compliant Motion Control, ASME Journal of Dynamic Systems, Measurement and Control, Vol 111(3), pp 416- 425, 1989.

Krebs, H. I., Aisen, M. L., Volpe, B. T. and Hogan, N., Robot Aided Neuro Rehabilitation: Initial Application to Stroke Rehabilitation, Proceedings of
MRCAS '95- 2nd International Symposium on Medical Robots and Computer Aided Surgery, John Wiley and Sons, Nov. '95.

Lum, Peter S., Lehman, Steven L. and Reinkensmeyer, David J., The Bimanual Lifting Rehabilitator: An Adaptive Machine for Therapy of Stroke Patients, IEEE Transactions on Rehabilitation Engineering, Vol. 3, No. 2, pp 166- 173, June 1995.

Lum, Peter S., Burgar, Charles G. and H. F. Machiel Van der Loos, The Use of a Robotic Device for Post Stroke Movement Therapy, Proceedings of the International Conference on Rehabilitation Robotics, Bath, U.K., April 14-15,1997, pp 79- 82.

McKormick, W. and Schwartz, H. M., An Investigation of Impedance Control for Robot Manipulators, International Journal of Robotics Research, Vol 12, No. 5, October 1993, pp 473- 489.

Noritsugu, T., Tanaka, T. and Yamanaka, T., Application of a Rubber Manipulator as a Rehabilitation Robot, IEEE International Workshop on Robot and Human Communication, pp 112- 117, 1996.

Poole, J.L. and Whitney, S.L., Motor assessment scale for stroke patients: concurrent validity and interrater reliability, Archives of Physical Medicine and Rehabilitation, 69, 195197, 1988. 
PUMA Mark II Robot 200 Series

Equipment Manual 1985.

Rao, R., A Robot Test-bed for Physical

Therapy, M.S. Thesis, Department of Mechanical Engineering, University of Delaware, 1990.

Rothstein, J.M., Measurement in Physical Therapy, New York: Churchill Livingstone, 1985.

Scholz J. P., Analysis of movement dysfunction: Control parameters and coordination stability, The 13th Annual Eugene Michels Researchers Forum, pp. 3-13. Alexandria, VA: American Physical Therapy Association, 1993.

Scholz, J. P. and Schoner, G., The uncontrolled manifold concept: identifying control variables for a functional task, In Press Experimental Brain Research, 1999.

Spong, Mark, W. and Vidyasagar, M., Robot Dynamics and Control, John Wiley and Sons, 1989.

Voss, D.E., Ionta, M.K. and Myers, B.J., Proprioceptive Neuromuscular Facilitation, Philadelphia, PA: Harper and Row Publishers, 1985.

Weinstein, C.J. Knowledge of results and motor learning - Implications for physical therapy, Physical Therapy, 71, 140- 149, 1990.

${ }^{\mathrm{i}}$ Graduate Student, Department of Mechanical Engineering

ii Assoc. Prof., Mechanical Engineering, Email: agrawal@me.udel.edu, Also, corresponding author

iii Associate Professor, Physical Therapy, Email:.jpscholz@udel.edu 